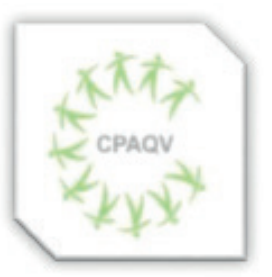

ISSN: 2178-7514

Vol. $13\left|\mathbf{N}^{\circ} .2\right|$ Ano 2021

\title{
COMPARAÇÃO DO ÂNGULO QUADRICIPITAL DE MEMBROS INFERIORES DOMINANTES E NÃO DOMINANTES EM UNIVERSITÁRIOS
}

Comparison of the quadricipital angle of lower dominant and non-dominant members in university students

Carlos Henrique Prevital Fileni ${ }^{1}$; Bráulio Nascimento Lima ${ }^{1,2}$; Gustavo Celestino Martins ${ }^{1,3}$; Leandro Borelli de Camargo ${ }^{1,3}$; José Ricardo Lourenço de Oliveira ${ }^{1}$; Klebson da Silva Almeida ${ }^{1,4}$; Marcelo Francisco Rodrigues ${ }^{1}$; Adriano de Almeida Pereira ${ }^{1}$; Luis Felipe Silio ${ }^{1,6}$; Ricardo Pablo Passos ${ }^{1,5}$; Guanis de Barros Vilela Junior ${ }^{1}$

\section{RESUMO}

O ângulo Q é uma medida importante do alinhamento de membros inferiores; sendo formado pelo encontro de duas linhas, a primeiro tem sua origem na Crista Ilíaca Superior Anterior (CISA) e vai até o centro da patela, e a segunda que vai da tuberosidade tibial até o centro da patela. Objetivo: Comparar os valores do ângulo Q de membros inferiores dominantes e não dominantes de universitários. Métodos: Participaram do estudo 330 estudantes do curso de Educação Física de um centro universitário em Campinas, sendo, 177 homens e 153 mulheres, com idade entre 18 a 30 anos. Os voluntários foram solicitados a realizar chutes com três bolas de futebol, sendo, um chute em cada bola para determinar a perna dominante, após esse procedimento foi dado início a coleta de dados. Os ângulos Q foram medidos a partir de fotos obtidas com câmera Sony Cybershot DSC 6MP, estando o eixo ótico da mesma perpendicular ao plano frontal dos sujeitos que estavam na posição ortostática, descalços, com os pés direcionados no sentido ântero-posterior. O eixo óptico da câmera estava direcionado ao ponto médio da coxa dos mesmos. Os ângulos foram medidos em ferramenta disponível no software Adobe Photoshop CS3, sendo aferidos em graus. Foram obtidos os ângulos Q para a perna dominante e não-dominante. Resultados: A média do ângulo Q para homens com membro dominante e não dominante foram respectivamente $14,85^{\circ}$ e $15,28^{\circ}$. Para as mulheres a média foi de $19,51^{\circ} \mathrm{em}$ membro dominante e $19,91^{\circ} \mathrm{em}$ membro não dominante. Quando realizado o teste de Wilcoxon para comparar pernas dominantes e não dominantes para homens e mulheres foram encontradas diferenças estatisticamente significantes, sendo que, para os homens 3752,00 para $\mathrm{p}<0,001$ e para as mulheres foi 1,000 para $\mathrm{p}<0,001$ entre pernas dominante e não dominante. A única correlação significante foi encontrada entre membro dominante e não dominante para homens. Conclusão: A avaliação de desvios posturais de membros inferiores e especialmente do ângulo Q, através da filmografia pode ser realizada para sujeitos eutróficos e para amostras maiores, dada a inviabilidade de se utilizar técnicas como imagens de RX. Houve diferenças significantes para ambos os sexos em relação ao ângulo Q de membros dominantes e não dominantes. Tal fato, pode ser consequência da eventual perda do equilíbrio biomecânico funcional entre os segmentos. Mais estudos precisam ser realizados para a compreensão da complexa rede de variáveis que podem interferir nestes desvios.

Palavras-chave: ângulo quadricipital; análise postural; biomecânica

\section{ABSTRACT}

The $\mathrm{Q}$ angle is an important measure of lower limb alignment; being formed by the meeting of two lines, the first has its origin in the Superior Anterior Iliac Crest (CISA) and goes to the center of the patella, and the second that goes from the tibial tuberosity to the center of the patella. Objective: To compare the Q angle values of dominant and non-dominant lower limbs in college students. Methods: 330 students of the Physical Education course from a college in Campinas participated in the study, 177 men and 153 women, aged between 18 and 30 years. The volunteers were asked to perform kicks with three soccer balls, with one kick in each ball to determine the dominant leg, after this procedure, data collection was started. Q angles were measured from photos taken with a Sony Cybershot DSC 6MP camera, with its optical axis perpendicular to the frontal plane of the subjects who were in the standing position, barefoot, with their feet facing anteroposteriorly. The optical axis of the camera was directed to the midpoint of their thigh. The angles were measured using a tool available in the Adobe Photoshop CS3 software, being measured in degrees. Q angles for the dominant and non-dominant leg were obtained. Results: The mean Q angle for men with dominant and non-dominant limbs were respectively $14.85^{\circ}$ and $15.28^{\circ}$. For women, the mean was $19.51^{\circ}$ in the dominant limb and $19.91^{\circ}$ in the non-dominant limb. When the Wilcoxon test was performed to compare dominant and non-dominant legs for men and women, statistically significant differences were found, with 3752.00 for men for $\mathrm{p}<0.001$ and for women it was 1.000 for $\mathrm{p}<0.001$ between dominant and non-dominant. The only significant correlation was found between dominant and non-dominant limb for men. Conclusion: The evaluation of postural deviations of the lower limbs, and especially of the Q angle, through filmography can be carried out for eutrophic subjects and for larger samples, given the infeasibility of using techniques such as X-ray images. There were significant differences for both sexes regarding the Q angle of dominant and non-dominant limbs. This fact may be a consequence of the eventual loss of the functional biomechanical balance between the segments. More studies need to be carried out to understand the complex network of variables that can interfere with these deviations.

Keywords: quadriceps angle; posture analysis; biomechanics

1 - Núcleo de Pesquisas em Biomecânica Ocupacional e Qualidade de Vida (NPBOQV - UNIMEP)

2 - Faculdade Conhecimento e Ciência - FCC

3 - Faculdade Euclides da Cunha - FEUC

4 - Universidade da Amazônia - UNAMA

5 - Anhanguera Sumaré

6 - Faculdade São Lucas Porto Velho

\section{Autor de correspondência}

Carlos Henrique Prevital Fileni

email: chpfileni@gmail.com 


\section{INTRODUÇÃO}

O Ângulo do Quadríceps (Ângulo Q) foi descrito pela primeira vez por Brattstroem ${ }^{(1)}$. Este ângulo é formado pelo encontro de duas linhas, a primeiro tem sua origem na Crista Ilíaca Superior Anterior (CISA) e vai até o centro da patela, e a segunda que vai da tuberosidade tibial até o centro da patela ${ }^{(2-4)}$.

A figura 1 mostra, esquematicamente, o ângulo Q médio para homens (A) e mulheres (B).

Figura 1 - Ângulo Q Médio para homens (A) e mulheres(B).

Figura 1 - Ângulo Q Médio para homens (A) e mulheres(B).

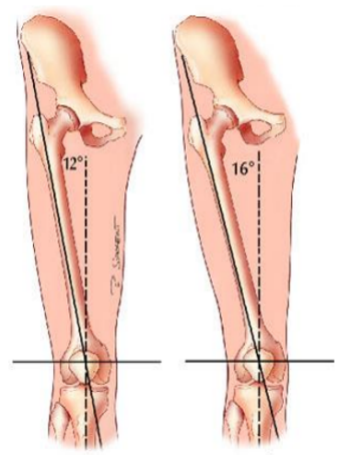

A - Homens

B - Mulheres

Powers ${ }^{(5)}$ indica que o Ângulo Q pode ser influenciado por três movimentos de membros inferiores, sendo eles: a rotação femoral, a rotação tibial e o desvio valgum. $\mathrm{O}$ aumento dessa rotação femoral pode resultar em um ângulo aumentado, logo que, a patela possui sua movimentação medialmente em relação à CISA. A rotação tibial em relação ao fêmur, tem a rotação lateral da tíbia deslocando a tuberosidade tibial para os lados, aumentando o Ângulo Q. Também, o valgum pode resultar na adução femoral, que dispõe a patela medialmente em relação à CISA, da abdução tibial, que desloca lateralmente a tuberosidade tibial ou de uma junção destes fatores.

A avaliação do Ângulo Q vem sendo analisada por diversos pesquisadores $(2-4,6,7) \mathrm{em}$ estudos sobre biomecânica, clínica e cirurgia do joelho. Esse ângulo contribui para determinar o vetor força que atua na patela e na articulação patelofemoral, identificando assim, preditores de risco de lesão.

O ângulo Q passou a ser aceito como um fator importante na avaliação da função articular do joelho ${ }^{(7)}$. Segundo Devan et al., ${ }^{(8)}$ alterações no ângulo Q em valgum alteram a biomecânica e prejudicam as alavancas musculares e, consequentemente, suas funções. Neste sentido, análise da relação entre Índice de Massa Corporal com o Ângulo Quadricipital faz-se necessário para se obter parâmetros biomecânicos, contribuindo assim, com o desempenho esportivo e prevenção de lesões.

Segundo Vilela Junior ${ }^{(9)}$ o ângulo Q funcional para homens na posição ortostática com os pés posicionados para frente e na largura dos ombros oscila entre $11^{\circ}$ e $17^{\circ}$; ao passo que para as mulheres o mesmo está entre $14^{\circ}$ e $20^{\circ}$.

Devido à grande importância clínica e biomecânica da avaliação do ângulo Q, o objetivo deste estudo foi comparar os valores do ângulo Q de membros inferiores dominantes e não dominantes de universitários. 


\section{MÉTODOS}

\section{DESENHO DO ESTUDO}

Trata-se de um estudo transversal observacional conduzido em Campinas, SP. Este trabalho foi submetido ao de Comitê de Ética e Pesquisa da Universidade Metodista de Piracicaba - UNIMEP, via Plataforma Brasil, aprovado com o protocolo 4.138.110, de acordo com a resolução $466 / 12$

\section{AMOSTRA}

Participaram do estudo 330 estudantes do curso de Educação Física de um centro universitário em Campinas, sendo, 177 homens e 153 mulheres, com idade entre 18 a 30 anos. A faixa etária foi escolhida de forma a excluir possíveis alterações degenerativas do joelho e quadril ${ }^{(10)}$. Os participantes foram recrutados por meio de comunicação pessoal, nas disciplinas de Cinesiologia, Fundamentos da Biomecânica e Biomecânica dos Esportes.

Critérios de inclusão: graduandos com idades $\geq 18$ anos de ambos os sexos; fisicamente normal; fisicamente ativos segundo critérios da Organização Mundial da Saúde.

Critérios de exclusão: graduandos que foram submetidos a cirurgia anterior nos joelhos, quadris, tornozelos e / ou coluna vertebral; histórias de luxação patelar; lesões meniscais ou outras lesões intra-articulares; evidência de edema; osteoartrite; anormalidades estruturais na coluna; discrepância de comprimento da perna de $2 \mathrm{~cm}$, avaliada por meio de medida real e aparente; envolvimento neurológico que comprometia a capacidade de andar; ou lesões musculares ou articulares no quadril.

Antes de iniciar o estudo, todos os sujeitos foram informados sobre os procedimentos da pesquisa, responderam a uma anamnese pessoal e assinaram um Termo de Consentimento, Livre e Esclarecido (TCLE).

\section{DELINEAMENTO ESPERIMENTAL}

Os voluntários foram solicitados a realizar chutes com três bolas de futebol, sendo, um chute em cada bola para determinar a perna dominante, após esse procedimento foi dado início a coleta de dados.

Para determinação do IMC foram coletados dados referentes a estatura utilizandose um estadiômetro da marca Sanny ${ }^{\circledR}$ graduado em centímetros. Para obtenção da massa corporal foi utilizado uma balança da marca Filizola ${ }^{\circledR} \mathrm{com}$ precisão de 100g. Para o cálculo do IMC foi utilizado a formula: IMC $=($ Massa corporal $) /$ (Estatura) $)^{2}$.

\section{MEDIÇÃO DO ÂNGULO Q}

Os ângulos $Q$ foram medidos a partir de fotos obtidas com câmera Cybershot Sony, estando o eixo ótico da mesma perpendicular ao plano frontal dos sujeitos que estavam na posição ortostática, descalços, com os pés direcionados no sentido ântero-posterior. O eixo óptico da câmera estava direcionado ao ponto médio da coxa dos mesmos. Para obtenção de cada foto os sujeitos foram orientados a trajarem roupas adequadas para a natação com a marcação 
dos pontos anatômicos de interesse (CISA. Ponto central da patela e tuberosidade tibial). Posteriormente, os ângulos foram medidos em ferramenta disponível no software Adobe Photoshop CS3, sendo aferidos em graus. Foram obtidos os ângulos Q para a perna dominante e não-dominante.

\section{ANÁLISE ESTATÍSTICA}

Os dados coletados alimentaram uma planilha no programa Microsoft Office Excel $2019^{\circledR}$ e analisados por meio do programa estatístico Statistical Package for the Social Sciences - SPSS, versão 25 para Windows, utilizando os seguintes testes: Kolmogorov-
Smirnov para testar a distribuição dos dados. As distribuições encontradas não foram normais, portanto, as comparações foram realizadas através do teste não paramétrico de Wilcoxon e a correlação de Spearman. Foram calculados o tamanho do efeito pela formula de Cohen. A significância adotada para este estudo foi de $\mathrm{p}<0,05$.

\section{RESULTADOS E DISCUSSÃO}

A seguir será apresentado os resultados referentes ao estudo e suas implicações no cotidiano.

Tabela 1 - Análise estatística descritiva

\begin{tabular}{lcccc}
\hline & Média & Desvio Padrão (DP) & Mínimo & Máximo \\
\cline { 2 - 5 } Idade_H (anos) & 22,05 & $\pm 2,63$ & 18 & 26 \\
Idade_M (anos) & 21,73 & $\pm 2,12$ & 18 & 28 \\
IMC_H (Kg/m²) & 23,50 & $\pm 1,48$ & 21,63 & 25,75 \\
IMC_M (Kg/m & 23,27 & $\pm 1,89$ & 20,40 & 26,52 \\
Ang_Q_H_D (graus) & 14,85 & $\pm 1,90$ & 9,30 & 18,50 \\
Ang_Q_H_ND (graus) & 15,28 & $\pm 1,81$ & 10,00 & 18,10 \\
Ang_Q_M_D (graus) & 19,51 & $\pm 3,52$ & 13,52 & 24,96 \\
Ang_Q_M_ND (graus) & 19,91 & $\pm 3,59$ & 13,80 & 26,00 \\
\hline IMC_H: İndice de Massa Corporal Homens; IMC_M: İndice de Massa Corporal Mulheres; Ang_Q_H_D: Ângulo Q \\
Homens Dominante; Ang_Q_H_ND: ÂnguloQ QHomens Não Dominante; Ang_QM_D: Ângulo QMulheres Dominante; \\
Ang_Q_M_ND: ÂnguloQQMulheres Não Dominante.
\end{tabular}

Fonte: Próprio autor, 2021

O ângulo Q é a medida de alinhamento patelar global, sendo seu valor normal, em média, de $13^{\circ}$ nos homens e $18^{\circ}$ nas mulheres ${ }^{(11-14)}$. Neste estudo a média do ângulo Q para homens com membro dominante e não dominante foram respectivamente $14,85^{\circ}$ e $15,28^{\circ}$, nota-se que o membro dominante está mais próximo do ângulo considerado normal, isso pode ser consequência de um melhor desenvolvimento muscular e articular decorrentes de uma predominância em tarefas cotidianas e esportivas. Para as mulheres os resultados encontrados não estão dentro dos considerados "ângulos normais" e destacase uma grande variação o mínimo e o máximo, especialmente, e membros não dominantes. 
Tabela 2 - Amostras Relacionadas de Resumo de Teste de Posto Sinalizado de Wilcoxon

\begin{tabular}{lclc}
\hline $\mathbf{N}^{0}$ total Homens & 177 & $\mathrm{~N}^{0}$ total Mulheres & 153 \\
Estatística do teste & 10794,000 & Estatística do teste & 11780,000 \\
Desvio Padrão & 642,101 & Desvio Padrão & 548,445 \\
$\begin{array}{l}\text { Estatística de Teste } \\
\text { Padronizado }\end{array}$ & 5,492 & $\begin{array}{l}\text { Estatística de Teste } \\
\text { Padronizado }\end{array}$ & 10,739 \\
$\begin{array}{l}\text { Sinal assintótico } \\
\text { (teste de dois lados) }\end{array}$ &, 000 & $\begin{array}{l}\text { Sinal assintótico (teste de } \\
\text { dois lados) }\end{array}$ &, 000 \\
Fonte: Próprio autor, 2021 & & &
\end{tabular}

Quando realizado o teste de Wilcoxon para comparar pernas dominantes e não dominantes para homens e mulheres foram encontradas diferenças estatisticamente significantes para ambos os sexos, sendo que, para os homens o resultado do referido teste foi de 3752,00 para $\mathrm{p}<0,001$ e para as mulheres foi de 1,000 para $\mathrm{p}<0,001$ entre pernas dominante e não dominante.

A seguir serão apresentadas as correlações de Spearman entre membro inferior dominante e não dominante de homens e mulheres.

Tabela 3 - Correlação Homens

\begin{tabular}{lcccc}
\hline & & & Ang_Q_H_D & Ang_Q_H_ND \\
\hline rô de & Ang_Q_H_D & Coeficiente de Correlaçãa & $1,000^{* *}$ &, $556^{* *}$ \\
\cline { 2 - 5 } Spearman & Ang_Q_H_ND & Coeficiente de Correlação &, $556^{* *}$ & $1,000^{* *}$
\end{tabular}

P<0,05**; IMC_H: İndice de Massa Corporal Homens; Ang_Q_H_D: Ångulo Q Homens Dominante; Ang_Q_H_ND: Ângulo Q Homens Não Dominante. Fonte: Próprio autor, 2021

Na tabela 3, nota-se que há correlação estatisticamente significante entre a perna dominante com a perna não dominante de homens, para as variáveis idade e IMC não teve correlação.

Os resultados do presente estudo corroboram com os achados por Maha et al., (3) onde, os autores buscaram comparar o ângulo Q entre os membros inferiores com relação a dominância, medindo a diferença entre os ângulos atléticos e não atléticos, explorando as variações dessas características em diferentes esportes. O estudo identificou uma diferença significante entre os grupos de atletas e não atletas. Da mesma forma, foi encontrada diferença estatisticamente significante no ângulo Q entre os membros inferiores em relação à dominância $(p<0,05)$. Não foram encontradas correlações significantes entre as pernas dominante e não dominante em relação ao IMC e Idade.

Em relação as mulheres não foram encontradas correlações significantes entre perna dominante e não dominante; o mesmo ocorrendo com o IMC e Idade.

A determinação do ângulo $Q$ ainda é controversa na comunidade cientifica, uma 
vez que, não existe um método que possa ser considerado "padrão ouro", tal fato pode ser explicado pelas dificuldades de se obter imagens de RX de sujeitos em posição ortostática que consigam mostrar a CISA, a patela e a tuberosidade tibial na mesma imagem. Por outro lado, a determinação do ângulo Q por fotogrametria por apresentar limitações decorrentes da dificuldade da localização adequada dos pontos anatômicos de interesse, especialmente em sujeitos com IMC $>25,00 \mathrm{Kg} / \mathrm{m}^{2}$.

No estudo de Santos et al., ${ }^{(15)}$ avaliaram a confiabilidade das medidas angulares obtidas a partir da fotogrametria computadorizada e a goniometria. Os autores tiveram como resultado que ambos os instrumentos são confiáveis e aceitáveis, porém, a confiabilidade das medições depende principalmente da uniformização dos procedimentos. Considerações metodológicas relativas ao estabelecimento de confiabilidade e padronização da colocação dos marcadores se fazem necessárias, de modo a oferecer opções de avaliação ainda mais confiáveis para a prática clínica.

A utilização mecânica de membro inferior dominante foi evidenciada neste estudo como um indicador de ângulo $\mathrm{Q}$ próximo do considerado normal, outro fator importante é o equilíbrio biomecânico funcional entre capacidades de força de músculos dos membros inferiores, tensionamento otimizado dos ligamentos, especialmente os colaterais do joelho. $\mathrm{Na}$ pesquisa de Marangon e Damázio ${ }^{(16)}$, onde os autores buscaram avaliar o ângulo Q e a incidência de dor em praticantes de spinning em academias na cidade de Ubá - MG. Os resultados obtidos não demonstraram evidências significantes de dor e alterações no ângulo Q das praticantes de spinning, as mesmas não apresentaram alterações do ângulo Q e incidência de dor nos joelhos.

Estudos envolvendo assimetria postural são necessários para identificar alterações no ângulo Q, outras pesquisas em biomecânica do esporte corroboram para prevenção e desempenho esportivo a partir de indicadores mecânicos.

No estudo de Sanchez et al., ${ }^{(17)}$ teve como objetivo comparar o valor do ângulo Q em diferentes posições, nas rotações externa e interna dos membros inferiores. Os resultados das comparações mostraram diferença significante entre a posição ortostática com pés paralelos e ortostática com pés abduzidos do lado esquerdo para ambos os sexos $(p=0,000)$. Também encontraram diferença significante entre a posição supina e ortostática com os pés abduzidos e com os pés paralelos à esquerda $(\mathrm{p}=0,046)$ no sexo feminino. A partir desses resultados, os autores concluíram que existem diferenças significantes na posição ortostática com os pés abduzidos e paralelos à perna esquerda, e simetria entre os membros inferiores independente da rotação dos membros na postura supina.

Leite e Cavalcanti Neto ${ }^{(18)}$, realizaram um estudo com atletas de futebol de campo feminino, observou-se durante jogos e treinos 
em um período de seis meses que a entorse de tornozelo foi o trauma mais frequente, e que as atletas lesionadas apresentavam alterações posturais como, genu-varo ou valgo, anomalias do pé, aumento do ângulo Q e assimetria de membros inferiores. Desta forma, acredita-se que, alterações posturais podem aumentar o risco de lesões traumato-ortopédicas nesta prática esportiva.

Em estudo realizado por Belchior et al., (11) teve como objetivo verificar a diferença entre o ângulo quadricipital em indivíduos sintomáticos e assintomáticos em duas situações, com o quadríceps relaxado e em contração isométrica voluntária máxima, afim de contribuir na avaliação de pacientes com disfunção fêmur-patelar. Os resultados obtidos mostram diferenças significantes nos indivíduos em estado de relaxamento entre os grupos, logo em estado de contração isométrica voluntária do musculo quadríceps não houve diferenças significantes, ocorrendo redução do ângulo Q em ambos os grupos.

O aumento do ângulo Q é multifatorial, podendo estar associado ao valgum femoral, ao ângulo de anteversão do fêmur acentuado, ao desvio medial da patela, debilidade do músculo vasto medial, dentre outros, o que atesta a complexidade da variação do mesmo.

\section{CONCLUSÃO}

No escopo desta pesquisa foi utilizada a fotogrametria, uma vez que, todos os sujeitos eram eutróficos. Outro aspecto decisivo na escolha na mesma foi a impossibilidade da obtenção de 330 imagens de RX na posição ortostática.

As diferenças entre os ângulos quadricipitais da perna dominante e não dominante podem ser consequência do desequilíbrio biomecânico funcional que pode acometer o membro não dominante; não obstante, estudos com outras populações devem ser replicados para uma eventual corroboração desta tese.

\section{REFERÊNCIAS}

1. Brattström H. Shape of the intercondylar groove normally and in recurrent dislocation of patella: a clinical and $\mathrm{x}$-ray anatomical investigation. Acta Orthopaedica Scandinavica. 1964;35(sup68):1-148.

2. Khasawneh RR, Allouh MZ, Abu-El-Rub E. Measurement of the quadriceps (Q) angle with respect to various body parameters in young Arab population. PloS one. 2019;14(6):e0218387.

3. El Gharib MH, El Tohamy AM, Mohamed NE. Determining the relationship between the quadriceps and tibiofemoral angles among adolescents. Journal of Taibah University Medical Sciences. 2021;16(1):70-6.

4. Heiderscheit BC, Hamill J, Van Emmerik R. Q-angle influences on the variability of lower extremity coordination during running. Medicine and Science in Sports and Exercise. 1999;31(9):1313-9.

5. Powers CM. The influence of altered lowerextremity kinematics on patellofemoral joint dysfunction: a theoretical perspective. Journal of Orthopaedic \& Sports Physical Therapy. 2003;33(11):639-46.

6. Almeida GPL, Carvalho APdMC, França FJR, Magalhães MO, Burke TN, Marques AP. Q-angle in patellofemoral pain: relationship with dynamic knee valgus, hip abductor torque, pain and function. Revista Brasileira de Ortopedia (English Edition). 2016;51(2):181-6.

7. Emami M-J, Ghahramani M-H, Abdinejad F, Namazi H. Q-angle: an invaluable parameter for evaluation of anterior knee pain. 2007.

8. Devan MR, Pescatello LS, Faghri P, Anderson J. A prospective study of overuse knee injuries among female athletes with muscle imbalances and 
structural abnormalities. Journal of athletic training. 2004;39(3):263.

9. Vilela Júnior G. Ângulo Q e os desvios Valgum

e Varum 2021. Available from: http://www.cpaqv.org/ cinesiologia/anguloq.pdf.

10. Russell KA, Palmieri RM, Zinder SM, Ingersoll CD. Sex differences in valgus knee angle during a single-leg drop jump. Journal of athletic training. 2006;41(2):166.

11. Belchior A, Arakaki J, Bevilaqua-Grossi D, Reis F, Carvalho P. Efeitos na medida do ângulo Q com a contração isométrica voluntária máxima do músculo quadricipital. Revista Brasileira de Medicina do esporte. 2006;12(1):6-10.

12. Nissen CW, Cullen MC, Hewett TE, Noyes FR. Physical and arthroscopic examination techniques of the patellofemoral joint. Journal of Orthopaedic \& Sports Physical Therapy. 1998;28(5):277-85.

13. Livingston LA. The quadriceps angle: a review of the literature. Journal of Orthopaedic \& Sports Physical Therapy. 1998;28(2):105-9.

14. Csintalan RP, Schulz MM, Woo J, McMahon PJ, Lee TQ. Gender differences in patellofemoral joint biomechanics. Clinical Orthopaedics and Related Research®. 2002;402:260-9.

15. Santos JDMd, Oliveira MAd, Silveira NJFd, Carvalho SdS, Oliveira AG. Confiabilidade inter e intraexaminadores nas mensurações angulares por fotogrametria digital e goniometria. Fisioterapia em Movimento. 2011;24(3):389-400.

16. Marangon MdSD, Laila Cristina Moreira Avaliação do ângulo Q e incidência de dor em praticantes de spinning em academias na Cidade de Ubá, MG. EFDeportescom, Revista Digital Buenos Aires, Año 15, $\mathrm{N}^{\circ}$ 152. 2011.

17. Sanchez HM, Sanchez EGdM, Baraúna MA, Canto RSdT. Evaluation of Q angle in differents static postures. Acta ortopedica brasileira. 2014;22(6):325-9.

18. Leite CBS, NETO FF. Incidência de lesões traumato-ortopédicas no futebol de campo feminino e sua relação com alterações posturais. Revista Digital. 2003;9(61).

OBSERVAÇÃO: Os autores declaram não existir conflitos de interesse de qualquer natureza. 